\title{
Depletion of the enteric nervous system by gene targeting results in haemorrhagic necrosis of the ileum
}

Bush TG, Savidge TC, Freeman TC, et al. Fulminant jejuno-ileitis following ablation of enteric glia in adult transgenic mice. Cell 1998;93:189-201.

\begin{abstract}
To investigate the roles of astroglial cells, we targeted their ablation genetically. Transgenic mice were generated expressing herpes simplex virus thymidine kinase from the mouse glial fibrillary acidic protein (GFAP) promoter. In adult transgenic mice, 2 weeks of subcutaneous treatment with the antiviral agent ganciclovir preferentially ablated transgene-expressing, GFAP-positive glia from the jejunum and ileum, causing a fulminating and fatal jejuno-ileitis. This pathology was independent of bacterial overgrowth and was characterized by increased myeloperoxidase activity, moderate degeneration of myenteric neurons, and intraluminal hemorrhage. These findings demonstrate that enteric glia play an essential role in maintaining the integrity of the bowel and suggest that their loss or dysfunction may contribute to the cellular mechanisms of inflammatory bowel disease.
\end{abstract}

\section{Comment}

Over the past few years it has become increasingly clear that new insights into the physiology of the gut are being made by non-gastroenterologists, often working on "bluesky" projects with no clinical relevance. One such project, published in Cell earlier this year, illustrates this point well.

Astrocytes are cells with branching cytoplasmic processes which surround nerves. Some of the processes are directed towards capillaries and others towards neurones. They provide structural support for the neurone, play a role in maintaining the blood-brain barrier, and are also important in repair after injury. They contain a characteristic protein-glial fibrillary acidic protein (GFAP). Bush and colleagues used the GFAP promoter to target the thymidine kinase of herpes simplex virus (HSV-TK) into astrocytes in transgenic mice. In proliferating cells exposed to the antiviral agent ganciclovir (GCV), HSV-TK metabolises GCV to produce toxic nucleotides which kill the cells expressing the transgene. Untreated transgenic mice are normal. However, when GCV was given to the transgenic mice in subcutaneous miniosmotic pumps, starting at about two weeks after implantation, the mice began to die. By 20 days treatment mortality was $100 \%$.

When the mice were analysed, the cause of death was clear - they all had a fulminant haemorrhagic jejuno-ileitis. The colon was normal. Time course studies showed that there was bleeding into the gut after 11 days of treatment. There were microthrombi in the vessels in the villi, haemorrhage and necrosis of villi, and large linear ulcers, extending into the deeper layers of the gut wall. There was a large increase in neutrophil myeloperoxidase in diseased mucosa, probably secondary to the loss of mucosal barrier function. Immunohistochemistry showed that GCV treatment produced a dramatic loss of GFAP+ glial cells in the lamina propria and submucosa, with the most severe loss in the areas of greatest tissue injury. Analysis of enteric neurones showed a less dramatic picture, but there was atrophy and loss of myenteric neurones. To investigate the role of the bacterial flora, some GCV treated transgenic mice were given antibiotics. This had no effect on the development of disease, although the experiment was not a great success because the antibiotics used had little effect on anaerobes.

One of the great surprises of the study was the fact that astrocyte loss was restricted to the small bowel and was not seen in the stomach, oesophagus or colon. In addition, there was no depletion of cells elsewhere in the nervous system. This is probably because the small bowel mucosal nervous system, like the rest of the mucosa, has a high tunover rate and there is continual remodelling and division of astrocytes, resulting in high expression of HSV-TK. ${ }^{1}$ However, the mechanisms by which loss of enteric nerves leads to such a profound phenotype in the small bowel can only be speculated upon. One rather obvious candidate is nitric oxide (NO) which is present in the neurones of the myenteric plexus, the submucosa, and muscularis mucosa but is rarely seen in the nerve fibres of the mucosa. ${ }^{2} \mathrm{NO}$ is a potent smooth muscle cell relaxant and it is conceivable that loss of NO secondary to depletion of neurones in the myenteric plexus causes contraction and narrowing of blood vessels, thus disrupting blood flow deep in the mucosa and making all of the changes seen in the mucosa secondary. Inhibition of NO in fetal sheep reduces blood flow and oxygen delivery to the small bowel, but not the colon. ${ }^{3}$ In addition, in rodents, mucosal blood flow is higher in the ileum than in the proximal bowel. ${ }^{4}$ Although Bush and colleagues seem to favour mucosal changes as being early events in the gut injury seen in these mice, their description of the pathology and some of the figures shown clearly indicate severe injury deep within the gut wall. Conversely, there was almost complete loss of glial cells from nerve fibres in the lamina propria and it is conceivable that the loss of glial cells disrupted neuropeptide production which synergised with the decreased NO to reduce drastically mucosal blood flow.

These studies may also be of clinical relevance. It has long been recognised that there is damage to nerves in Crohn's disease, ${ }^{5}$ hardly surprising given the transmural nature of the inflammation. Although these changes are almost certainly secondary, once established, they may functionally compromise neuronal control of blood flow to the gut mucosa, predisposing to ischaemia and scarring in long-standing disease. Finally, Bush and colleagues speculate that the lesions seen in the GCV treated mice resemble Crohn's disease, but the micrographs supplied were not persuasive that this was the case. This, however, is a trivial point compared with the broad lesson to be learned from the fact that mice with disrupted epithelial integrity, disrupted immunoregulatory cytokine genes or immu- 
noregulatory cells, or altered thymocyte development (reviewed by Simpson and colleagues ${ }^{6}$ ), and now a disrupted enteric nervous system, all develop inflammatory bowel disease. There clearly are many routes which can produce an inflammatory bowel disease phenotype, something gastroenterologists should take on board the next time they hear a talk from someone claiming to have found the cause of Crohn's disease.

T T MacDONALD

Department of Paediatric Gastroenterology, St Bartholomew's Hospital, Bartholomew Close, London EC1A 7BE, UK email:t.t.macdonald@mds.qmw.ac.uk
1 Sharkey KA, Coggins PJ, Tetzlaff W, et al. Distribution of growth associated protein B-50 (GAP-43) in the mammalian enteric nervous system. Neuroscience 1990;38:13-20.

2 De Giorgio R, Parodi JE, Brecha NC, et al. Nitric oxide producing neurones in the monkey and human digestive system. F Comp Neurol 1994;342:61927.

3 Fan WQ, Smolich JJ, Wild J, et al. Nitric oxide modulates regional blood flow differences in the fetal gastrointestinal tract. Am $\mathcal{f}$ Physiol 1996;271:G598-604.

4 Klemm K, Moody FG. Regional intestinal blood flow and nitric oxide synthase inhibition during sepsis in the rat. Ann Surg 1998;227:126-33.

5 Kubota Y, Petras RE, Ottaway CA, et al. Colonic vasoactive intestinal peptide nerves in inflammatory bowel disease. Gastroenterology 1992;102: $1242-51$

6 Simpson SJ, de Jong YP, Comiskey M, et al. T cells in mouse models of gut inflammation. In: MacDonald TT, ed. Mucosal T cells. Basel: Karger, 1998: $118-38$. 